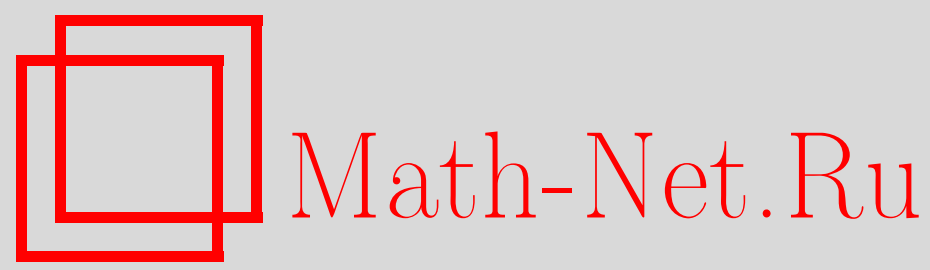

С. Ю. Тихонов, Об интегрируемости тригонометрических рядов, Матем. заметки, 2005, том 78, выпуск 3, 476-480

DOI: https://doi.org/10.4213/mzm2606

Использование Общероссийского математического портала Math-Net.Ru подразумевает, что вы прочитали и согласны с пользовательским соглашением http://www. mathnet.ru/rus/agreement

Параметры загрузки:

IP: 3.81 .55 .215

26 апреля 2023 г., 15:19:28

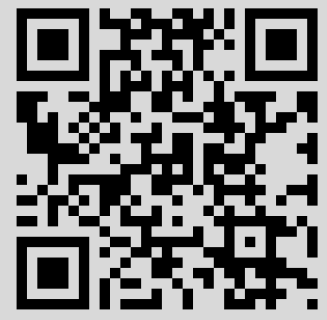




\section{ОБ ИНТЕГРИРУЕМОСТИ ТРИГОНОМЕТРИЧЕСКИХ РЯДОВ}

\section{С. Ю. Тихонов}

1. Введение. В работе изучаются вопросы интегрируемости рядов $g(x)=\sum_{n=1}^{\infty} \lambda_{n} \sin n x$ и $f(x)=\sum_{n=1}^{\infty} \lambda_{n} \cos n x$. Прежде всего, запишем классический результат Янга-Боаса-Хейвуда для рядов с монотонно убывающими коэффициентами.

TeOpema 1 [1]-[3]. Пусть $\lambda_{n} \downarrow 0$. Ecлu $0 \leqslant \alpha<2$, mo

$$
\frac{g(x)}{x^{\alpha}} \in L(0, \pi) \Longleftrightarrow \sum_{n=1}^{\infty} n^{\alpha-1} \lambda_{n}<\infty .
$$

Eсли $0<\alpha<1$, mo

$$
\frac{f(x)}{x^{\alpha}} \in L(0, \pi) \Longleftrightarrow \sum_{n=1}^{\infty} n^{\alpha-1} \lambda_{n}<\infty .
$$

В дальнейших исследованиях этот результат обобщался в нескольких направлениях (см., например, $[4],[5])$ : рассматривались более общие весовые функции $\gamma$, рассматривалась интегрируемость с весом $\gamma$ функций $g(x)$ и $f(x)$ в степени $p$ для разных значений $p$, также рассматривались более общие условия на коэффициенты $\left\{\lambda_{n}\right\}$.

Запишем следуюшее определение (см. [6]). Нуль-последовательность положительных чисел с := $\left\{c_{n}\right\}$ принадлежит классу $R_{0}^{+} B V S$, если неравенство $\sum_{n=m}^{\infty}\left|c_{n}-c_{n+1}\right| \leqslant K(\mathbf{c}) c_{m}$ справедливо для всех натуральных $m$. Ясно, что класс монотонных последовательностей $M=\left\{\mathbf{c}: c_{n} \downarrow 0\right\}$ есть подк ласс $R_{0}^{+} B V S$, но класс квазимонотонных последовательностей $Q M=\{\mathbf{c}: \exists \tau \geqslant 0$ такое, что $\left.c_{n} n^{-\tau} \downarrow 0\right\}$ не сравним с классом $R_{0}^{+} B V S$ (см. [6]), т.е. найдется с ${ }^{*} \in Q M$, но $\mathbf{c}^{*} \notin R_{0}^{+} B V S$, и наоборот.

В [6] показано, что ряды с коэффициентами из класса $R_{0}^{+} B V S$ сходятся, кроме, возможно, $x=0$ для косинус-ряда и сходятся равномерно на промежутке $\delta \leqslant x \leqslant \pi-\delta$ для любого $0<\delta<\pi$. Там же доказана следующая

Teоpema 2. Пусmb $\left\{\lambda_{n}\right\} \in R_{0}^{+} B V S, 1<p<\infty u 1 / p-1<\theta<1 / p$. Тогдa

$$
\frac{|\psi(x)|^{p}}{x^{p \theta}} \in L(0, \pi) \Longleftrightarrow \sum_{n=1}^{\infty} n^{p \theta+p-2} \lambda_{n}^{p}<\infty,
$$

где функиия $\psi(x)-$ или $f(x)$, или $g(x)$.

Недавно в [7] были рассмотрены более общие, чем степенные, весовые функции и найдены достаточные условия интегрируемости с такими весами синус-рядов.

Теорема 3. Пусть $\left\{\lambda_{n}\right\} \in R_{0}^{+}$BVS и последовательность $\gamma:=\left\{\gamma_{n}\right\}$ удовлетворяет условию: существует $\varepsilon>0$ такое, что последовательность $\left\{\gamma_{n} n^{-2+\varepsilon}\right\}$ почти убивает. Тогда

$$
\sum_{n=1}^{\infty} \frac{\gamma_{n}}{n} \lambda_{n}<\infty \Longrightarrow \gamma(x) g(x) \in L(0, \pi) .
$$

Здесь и далее мы определяем функцию $\gamma(x)$ по последователности $\gamma$ следующим образом: $\gamma(\pi / n):=\gamma_{n}, n \in \mathbb{N}$, и существуют положительные константы $A$ и $B$ такие, что

Работа выполнена при частичной поддержке Российского фонда фундаментальных исследований, грант № 03-01-00080, European Commission, Contract MIF1-2004-509465, и Centre de Recerca Matematica, Barcelona. 
$A \gamma_{n} \leqslant \gamma(x) \leqslant B \gamma_{n+1}$ для $x \in(\pi /(n+1), \pi / n)$. Последовательность положительных чисел $\gamma:=\left\{\gamma_{n}\right\}$ называется почти возрастающей (почти убыв ающей), если неравенство $C \gamma_{n} \geqslant \gamma_{m}$ $\left(\gamma_{n} \leqslant C \gamma_{m}\right)$ справедливо для всех натуральных $n \geqslant m$.

В данной работе решается следующая задача: найти необходимые и достаточные условия интегрируемости в $p$-й степени сумм синус- и косинус-рядов с весом $\gamma$. В качестве решения мы выпишем условия на коэффициенты $\left\{\lambda_{n}\right\}$, которые, в зависимости от поведения $\gamma$, будут достаточными или необходимыми. Полученные результаты уточняют результаты теорем 1-3.

\section{2. Основные результаты.}

Tеорема 4. Пусть $\left\{\lambda_{n}\right\} \in R_{0}^{+} B V S u 1 \leqslant p<\infty$.

А) Если последовательность $\left\{\gamma_{n}\right\}$ удовлетворяет условию: существует $\varepsilon_{1}>0$ такое, что последовательность $\left\{\gamma_{n} n^{-p-1+\varepsilon_{1}}\right\}$ является почти убьвающей, то условие

$$
\sum_{n=1}^{\infty} \gamma_{n} n^{p-2} \lambda_{n}^{p}<\infty
$$

достаточно для выполнения условия

$$
\gamma(x)|g(x)|^{p} \in L(0, \pi) .
$$

Б) Если последовательность $\left\{\gamma_{n}\right\}$ удовлетворяет условию: существует $\varepsilon_{2}>0$ такое, что последовательность $\left\{\gamma_{n} n^{p-1-\varepsilon_{2}}\right\}$ является почти возрастающ,й, то условие (1) необходимо для выполнения условия (2).

TEOPEMA 5. Пусть $\lambda_{n} \in R_{0}^{+} B V S u 1 \leqslant p<\infty$.

А) Если последовательность $\left\{\gamma_{n}\right\}$ удовлетворяет условию: существует $\varepsilon_{3}>0$ такое, что последовательность $\left\{\gamma_{n} n^{-1+\varepsilon_{3}}\right\}$ является почти убьвающ,еи, то условие

$$
\sum_{n=1}^{\infty} \gamma_{n} n^{p-2} \lambda_{n}^{p}<\infty
$$

достаточно для выполнения условия

$$
\gamma(x)|f(x)|^{p} \in L(0, \pi) .
$$

Б) Если последовательность $\left\{\gamma_{n}\right\}$ удовлетворяет условию: существует $\varepsilon_{4}>0$ такое, что последовательность $\left\{\gamma_{n} n^{p-1-\varepsilon_{4}}\right\}$ является почти возрастающей, то условие (3) необходимо для выполнения условия (4).

\section{3. Доказательство теорем.}

ДоКАЗАТЕЛЬСтво тЕОРемы 4. А) Пусть $x \in(\pi /(n+1), \pi / n]$. Тогда, используя преобразование Абеля, получим

$$
|g(x)| \leqslant x \sum_{k=1}^{n} k \lambda_{k}+\left|\sum_{k=n+1}^{\infty} \lambda_{k} \sin k x\right| \leqslant x \sum_{k=1}^{n} k \lambda_{k}+\sum_{k=n}^{\infty}\left|\left(\lambda_{k}-\lambda_{k+1}\right) \widetilde{D}_{k}(x)\right|,
$$

где $\widetilde{D}_{k}(x):=\sum_{n=1}^{k} \sin n x, k \in \mathbb{N}$. Используя соотношение $\left|\widetilde{D}_{k}(x)\right|=O(1 / x)$ и условие $\left\{\lambda_{n}\right\} \in$ $R_{0}^{+} B V S$, получим

$$
|g(x)| \leqslant C\left(x \sum_{k=1}^{n} k \lambda_{k}+n \sum_{k=n}^{\infty}\left|\lambda_{k}-\lambda_{k+1}\right|\right) \leqslant C\left(x \sum_{k=1}^{n} k \lambda_{k}+n \lambda_{n}\right) \leqslant \frac{C}{n} \sum_{k=1}^{n} k \lambda_{k} .
$$

В последнем неравенстве мы использовали тот факт, что если $\lambda=\left\{\lambda_{n}\right\} \in R_{0}^{+} B V S$, то $\lambda$ почти убывает. Здесь и далее $C$ и $C_{i}$ будут обозначать положительные константы, быть может, разные в разных случаях. 
Таким образом,

$$
\int_{0}^{\pi} \gamma(x)|g(x)|^{p} d x=\sum_{n=1}^{\infty} \int_{\pi /(n+1)}^{\pi / n} \gamma(x)|g(x)|^{p} d x \leqslant C \sum_{n=1}^{\infty} \frac{\gamma_{n}}{n^{2+p}}\left(\sum_{k=1}^{n} k \lambda_{k}\right)^{p} .
$$

Применяя (см., например, [8]) неравенство для последовательностей $\left\{a_{n} \geqslant 0\right\}$ и $\left\{\lambda_{n}>0\right\}$ :

$$
\sum_{n=1}^{\infty} \lambda_{n}\left(\sum_{\nu=1}^{n} a_{\nu}\right)^{p} \leqslant p^{p} \sum_{n=1}^{\infty} \lambda_{n}^{1-p} a_{n}^{p}\left(\sum_{\nu=n}^{\infty} \lambda_{\nu}\right)^{p}, \quad p \geqslant 1
$$

получим

$$
\int_{0}^{\pi} \gamma(x)|g(x)|^{p} d x \leqslant C \sum_{k=1}^{\infty}\left(k \lambda_{k}\right)^{p}\left(\frac{\gamma_{k}}{k^{2+p}}\right)^{1-p}\left[\sum_{\nu=k}^{\infty} \frac{\gamma_{\nu}}{\nu^{2+p}}\right]^{p} .
$$

Используя условия на последовательность $\left\{\gamma_{\nu}\right\}$, получим

$$
\sum_{\nu=k}^{\infty} \frac{\gamma_{\nu}}{\nu^{2+p}} \leqslant C \frac{\gamma_{k}}{k^{1+p-\varepsilon_{1}}} \sum_{\nu=k}^{\infty} \nu^{-\varepsilon_{1}-1} \leqslant C \frac{\gamma_{k}}{k^{1+p}}
$$

тогда

$$
\int_{0}^{\pi} \gamma(x)|g(x)|^{p} d x \leqslant C \sum_{k=1}^{\infty} \gamma_{k} k^{p-2} \lambda_{k}^{p}
$$

что завершает доказательство пункта А.

Б) Заметим, что из условия (2) следует, что $g(x) \in L(0, \pi)$. Действительно, если $p \in(1, \infty)$, то применяя неравенство Гёльдера, получим

$$
\int_{0}^{\pi}|g(x)| d x \leqslant\left(\int_{0}^{\pi}|g(x)|^{p} \gamma(x) d x\right)^{1 / p}\left(\int_{0}^{\pi}(\gamma(x))^{-p^{\prime} / p} d x\right)^{1 / p^{\prime}}=: I,
$$

где $p^{\prime}=p /(p-1)$. Так как

$$
\int_{0}^{\pi}(\gamma(x))^{-p^{\prime} / p} d x \leqslant C \sum_{n=1}^{\infty}\left(\frac{1}{\gamma_{n}}\right)^{1 /(p-1)} \int_{\pi /(n+1)}^{\pi / n} d x \leqslant\left(\frac{C}{\gamma_{1}}\right)^{1 /(p-1)} \sum_{n=1}^{\infty} n^{-1-\frac{\varepsilon_{2}}{p-1} \leqslant C_{1}}
$$

то из условия (2) следует $I \leqslant C_{2}$.

Пусть $p=1$. Тогда можно утверждать, что $\gamma_{n}$ почти возрастает и, значит,

$$
\int_{0}^{\pi}|g(x)| d x \leqslant \sum_{n=1}^{\infty} \frac{1}{A \gamma_{n}} \int_{\pi /(n+1)}^{\pi / n}|g(x)| \gamma(x) d x \leqslant \frac{C}{\gamma_{1}} \int_{0}^{\pi}|g(x)| \gamma(x) d x<C .
$$

В дальнейшем будем использовать условие $g(x) \in L(0, \pi)$. Интегрируя $g(x)$, получим

$$
F(x):=\int_{0}^{x} g(t) d t=\sum_{n=1}^{\infty} \frac{\lambda_{n}}{n}(1-\cos n x)=2 \sum_{n=1}^{\infty} \frac{\lambda_{n}}{n} \sin ^{2} \frac{n x}{2} .
$$

Используя тот факт, что последовательность $\left\{\lambda_{n}\right\}$ почти убьвает, получим

$$
F\left(\frac{\pi}{k}\right) \geqslant C \sum_{n=[k / 2]}^{k} \frac{\lambda_{n}}{n}\left(\frac{n}{k}\right)^{2} \geqslant C \frac{\lambda_{k}}{k^{2}} \sum_{n=[k / 2]}^{k} n \geqslant C \lambda_{k} .
$$


Обозначим $d_{\nu}:=\int_{\pi /(\nu+1)}^{\pi / \nu}|g(x)| d x, \nu \in \mathbb{N}$. Тогда $\lambda_{k} \leqslant C F(\pi / k) \leqslant C \sum_{\nu=k}^{\infty} d_{\nu}$. Далее запишем (см. [8]) неравенство $\left(\left\{a_{n} \geqslant 0\right\}\right.$ и $\left.\left\{\lambda_{n}>0\right\}\right)$

$$
\sum_{n=1}^{\infty} \lambda_{n}\left(\sum_{\nu=n}^{\infty} a_{\nu}\right)^{p} \leqslant p^{p} \sum_{n=1}^{\infty} \lambda_{n}^{1-p} a_{n}^{p}\left(\sum_{\nu=1}^{n} \lambda_{\nu}\right)^{p}, \quad p \geqslant 1,
$$

применяя которое, получим

$$
J:=\sum_{k=1}^{\infty} \gamma_{k} k^{p-2} \lambda_{k}^{p} \leqslant C \sum_{k=1}^{\infty} \gamma_{k} k^{p-2}\left(\sum_{\nu=k}^{\infty} d_{\nu}\right)^{p} \leqslant C \sum_{\nu=1}^{\infty} d_{\nu}^{p}\left(\gamma_{\nu} \nu^{p-2}\right)^{1-p}\left\{\sum_{k=1}^{\nu} \gamma_{k} k^{p-2}\right\}^{p} .
$$

По условию теоремы последовательность $\left\{\gamma_{n} n^{p-1-\varepsilon_{2}}\right\}$ почти возрастает. Тогда

$$
J \leqslant C \sum_{\nu=1}^{\infty} d_{\nu}^{p}\left(\gamma_{\nu} \nu^{p-2}\right)^{1-p}\left\{\gamma_{\nu} \nu^{p-1-\varepsilon_{2}} \sum_{k=1}^{\nu} k^{\varepsilon_{2}-1}\right\}^{p} \leqslant C \sum_{\nu=1}^{\infty} d_{\nu}^{p} \gamma_{\nu} \nu^{2(p-1)} .
$$

Если $p \in(1, \infty)$, то применяя неравенство Гёльдера, получим $\left(p^{\prime}=p /(p-1)\right)$

$$
\begin{aligned}
d_{\nu}^{p} & =\left(\int_{\pi /(\nu+1)}^{\pi / \nu}|g(x)| d x\right)^{p} \leqslant C \int_{\pi /(\nu+1)}^{\pi / \nu}|g(x)|^{p} d x\left(\frac{1}{\nu^{2}}\right)^{p / p^{\prime}} \\
& =C \nu^{2(1-p)} \int_{\pi /(\nu+1)}^{\pi / \nu}|g(x)|^{p} d x .
\end{aligned}
$$

Следовательно,

$$
J \leqslant C \sum_{\nu=1}^{\infty} \gamma_{\nu} \int_{\pi /(\nu+1)}^{\pi / \nu}|g(x)|^{p} d x \leqslant C \int_{0}^{\pi}|g(x)|^{p} \gamma(x) d x .
$$

Если $p=1$, то

$$
J \leqslant C \sum_{\nu=1}^{\infty} d_{\nu} \gamma_{\nu} \leqslant C \int_{0}^{\pi}|g(x)| \gamma(x) d x
$$

Теорема доказана.

ДоКАЗАТЕЛЬСтво тЕОРЕмЫ 5. Пункт А) доказывается так же, как и в теореме 4. Действительно, используя неравенство $|f(x)| \leqslant C \sum_{k=1}^{n} \lambda_{k}$ для $x \in(\pi /(n+1), \pi / n]$, неравенство (5) и условия на $\gamma$, получим

$$
\begin{aligned}
\int_{0}^{\pi} \gamma(x)|f(x)|^{p} d x & \leqslant C \sum_{n=1}^{\infty} \frac{\gamma_{n}}{n^{2}}\left(\sum_{k=1}^{n} \lambda_{k}\right)^{p} \\
& \leqslant C \sum_{k=1}^{\infty} \lambda_{k}^{p}\left(\frac{\gamma_{k}}{k^{2}}\right)^{1-p}\left[\sum_{\nu=k}^{\infty} \frac{\gamma_{\nu}}{\nu^{2}}\right]^{p} \leqslant C \sum_{k=1}^{\infty} \gamma_{k} k^{p-2} \lambda_{k}^{p}
\end{aligned}
$$

Б) Из условия (4) следует (аналогично доказательству $g(x) \in L(0, \pi)$ в теореме 4 ), что $f(x) \in$ $L(0, \pi)$. Интегрируя $f(x)$, запишем

$$
H(x)=\int_{0}^{x} f(t) d t=\sum_{n=1}^{\infty} \frac{\lambda_{n}}{n} \sin n x .
$$

Так как $\left\{\lambda_{n}\right\} \in R_{0}^{+} B V S$ влечет $\left\{\lambda_{n} / n\right\} \in R_{0}^{+} B V S$, то, применяя пункт Б) теоремы 4 для функции $H(x)$, получим

$$
\sum_{k=1}^{\infty} \gamma_{k}^{*} k^{p-2}\left(\frac{\lambda_{k}}{k}\right)^{p} \leqslant C \int_{0}^{\pi} \gamma^{*}(t)|H(t)|^{p} d t
$$


где $\left\{\gamma_{k}^{*}\right\}$ удовлетворяет условию: существует $\varepsilon>0$ такое, что последовательность $\left\{\gamma_{n}^{*} n^{p-1-\varepsilon}\right\}$ почти возрастает. Для $\left\{\gamma_{n}^{*}=\gamma_{n} n^{p}\right\}$ это условие, очевидно, выполнено. Тогда

$$
\sum_{k=1}^{\infty} \gamma_{k} k^{p-2} \lambda_{k}^{p} \leqslant C \int_{0}^{\pi} \frac{\gamma(x)}{x^{p}}|H(x)|^{p} d x \leqslant C \sum_{k=1}^{\infty} \gamma_{k} k^{p-2}\left(\sum_{\nu=k}^{\infty} \int_{\pi /(\nu+1)}^{\pi / \nu}|f(t)| d t\right)^{p} .
$$

Обозначая $f_{\nu}=\int_{\pi /(\nu+1)}^{\pi / \nu}|f(x)| d x, \nu \in \mathbb{N}$ и используя $(6)$, получим

$$
\sum_{k=1}^{\infty} \gamma_{k} k^{p-2} \lambda_{k}^{p} \leqslant C \sum_{k=1}^{\infty} \gamma_{k} k^{p-2}\left(\sum_{\nu=k}^{\infty} f_{\nu}\right)^{p} \leqslant C \sum_{\nu=1}^{\infty} f_{\nu}^{p}\left(\gamma_{\nu} \nu^{p-2}\right)^{1-p}\left\{\sum_{k=1}^{\nu} \gamma_{k} k^{p-2}\right\}^{p}
$$

Аналогично (7)-(10) для $p \geqslant 1$ можно записать

$$
\sum_{k=1}^{\infty} \gamma_{k} k^{p-2} \lambda_{k}^{p} \leqslant C \int_{0}^{\pi}|f(x)|^{p} \gamma(x) d x
$$

Теорема доказана.

\section{СПИСОК ЦИТИРОВАННОЙ ЛИТЕРАТУРЫ}

1. Young W. H. // Proc. London Math. Soc. 1913. V. 12. P. 41-70. 2. Boas Jr. R. P. // Quart. J. Math. Oxford Ser. (2). 1952. V. 3. P. 217-221. 3. Heywood P. // Quart. J. Math. Oxford Ser. (2). 1954. V. 5. P. 71-76. 4. Aljancic S., Bojanic R., Tomic M. // Acad. Serbe Sci., Publ. Inst. Math. 1955. V. 8. P. 67-84. 5. Igari S. // Tôhoku Math. J. 1960. V. 12. P. 139-146. 6. Leindler L. // Anal. Math. 2002. V. 28. №4. P. 279-286. 7. Németh J. // J. Inequal. Pure Appl. Math. 2003. V. 4. № 1. Article 3, 6 pp. 8. Потапов M. K., Бериша M. // Publ. Inst. Math. (Beograd) (N. S. ). 1979. T. 26(40). C. 215-228.

Московский государственный университет им. М.В. Ломоносова, 